\title{
Mutations in the human $\delta$-sarcoglycan gene in familial and sporadic dilated cardiomyopathy
}

\author{
Shinichi Tsubata, ${ }^{1,2}$ Karla R. Bowles, ${ }^{3}$ Matteo Vatta, ${ }^{2}$ Carmelann Zintz, ${ }^{2}$ Jack Titus, ${ }^{4}$ \\ Linda Muhonen, ${ }^{5}$ Neil E. Bowles, ${ }^{2}$ and Jeffrey A. Towbin ${ }^{2,3,6}$ \\ ${ }^{1}$ Department of Pediatrics, Toyama Medical and Pharmaceutical University, Toyama, Japan \\ ${ }^{2}$ Department of Pediatrics, Section of Cardiology, and \\ ${ }^{3}$ Department of Molecular and Human Genetics, Baylor College of Medicine, Houston, Texas, USA \\ ${ }^{4}$ The Jesse Edwards Cardiac Registry, Minneapolis, Minnesota, USA \\ ${ }^{5}$ Department of Pediatrics, Section of Cardiology, Orange County Children's Hospital, Orange, California, USA \\ ${ }^{6}$ Department of Cardiovascular Sciences, Baylor College of Medicine, Houston, Texas, USA
}

Address correspondence to: Jeffrey A. Towbin, Department of Pediatrics, Section of Cardiology, Baylor College of Medicine, One Baylor Plaza, Room 333E, Houston, Texas 77030, USA.

Phone: (713) 798-7342; Fax: (713) 798-8085; E-mail: jtowbin@bcm.tmc.edu.

Received for publication December 21, 1999, and accepted in revised form July 25, 2000.

Dilated cardiomyopathy (DCM) is a major cause of morbidity and mortality. Two genes have been identified for the X-linked forms (dystrophin and tafazzin), whereas three other genes (actin, lamin $A / C$, and desmin) cause autosomal dominant DCM; seven other loci for autosomal dominant DCM have been mapped but the genes have not been identified. Hypothesizing that DCM is a disease of the cytoskeleton and sarcolemma, we have focused on candidate genes whose products are found in these structures. Here we report the screening of the human $\delta$-sarcoglycan gene, a member of the dystrophinassociated protein complex, by single-stranded DNA conformation polymorphism analysis and by DNA sequencing in patients with DCM. Mutations affecting the secondary structure were identified in one family and two sporadic cases, whereas immunofluorescence analysis of myocardium from one of these patients demonstrated significant reduction in $\delta$-sarcoglycan staining. No skeletal muscle disease occurred in any of these patients. These data suggest that $\delta$-sarcoglycan is a disease-causing gene responsible for familial and idiopathic DCM and lend support to our "final common pathway" hypothesis that DCM is a cytoskeletalopathy.

J. Clin. Invest. 106:655-662 (2000).

\section{Introduction}

Dilated cardiomyopathy (DCM) is a primary heart muscle disease characterized by left ventricular dilation and systolic dysfunction, with secondary diastolic dysfunction and occasionally associated right ventricular disease (1). This disorder has an incidence of $3.5-8.5 / 100,000$ population per year and a prevalence of approximately $36 / 100,000$ population $(2,3)$, which appears to be on the rise. In addition, DCM is the most common reason for heart failure and for cardiac transplantation in the US, with an estimated cost of $\$ 10$ billion to $\$ 40$ billion yearly (4).

The underlying causes of DCM are heterogeneous (5, 6), including myocarditis, drug toxicity (adriamycin), and ischemia-induced, metabolic, mitochondrial, and genetic abnormalities. A genetic cause of DCM is identified in approximately $30 \%$ of cases (7-9), with autosomal dominant inheritance being the most common (6). Xlinked, autosomal recessive, and mitochondrial inheritance have also been reported, albeit less frequently (10).

In the past several years, the genetic basis of DCM has been sought, resulting in the identification of multiple genetic loci and five genes causing DCM to date. For Xlinked DCM, two genes have been identified, including tafazzin (G4.5) in cases of the infantile-onset DCM (Barth syndrome) $(11,12)$ and isolated left ventricular noncompaction $(13,14)$, and dystrophin in later-onset Xlinked cardiomyopathy (XLCM) (15-17). In the more common autosomal dominant DCM, five loci have been mapped for pure DCM (1q32 [ref. 18], 2q31 [ref. 19], 9q13-q22 [ref. 20], 10q21-q23 [ref. 21], and 15q14 [ref. 22]), and four loci have been mapped in families with DCM and associated with conduction disease (1p1-1q21 [ref. 23], 2q14-q22 [ref. 24], 2q35 [ref. 25], 3p25-p22 [ref. 26], and 6q23 [ref. 27]). Thus far, only the gene on chromosome 15q14 encoding cardiac actin (22), the gene on chromosome $2 \mathrm{q} 35$ encoding desmin (25), and the gene on chromosome 1p1-1q21 encoding lamin $A / C$ (28) have been identified and mutations characterized.

We have proposed a "final common pathway" hypothesis $(29,30)$, which states that hereditary cardiovascular diseases with similar phenotypes and genetic heterogeneity will occur due to abnormalities in genes encoding proteins of similar function or genes encoding proteins participating in a common pathway cascade. In the case of DCM, we have proposed that mutations affect elements of the cell cytoarchitecture, based around the cytoskeleton (i.e., a cytoskeletalopathy) and sarcolemma, but also including elements that interact with the cytoskeleton including dystrophin, 
the dystrophin-associated glycoprotein complex (DAG; i.e., the sarcoglycans and dystroglycans), and intermediate filaments. Other final common pathways for specific cardiac phenotypes include the sarcomere in hypertrophic cardiomyopathy (31) and ion channels in cardiac rhythm disorders, such as long QT syndromes and Brugada syndrome (32).

Animal models of cardiomyopathy have also been described, including the BIO14.6 hypertrophic cardiomyopathy and the TO-2 DCM hamsters $(33,34)$, which are both due to mutations in the $\delta$-sarcoglycan gene (35-37). These animals also exhibit histological features of muscular dystrophy (38). Mutations in this gene in humans cause autosomal recessive limbgirdle muscular dystrophy, LGMD2F (39), a disorder in which skeletal muscle disease can vary from mild (i.e., ambulatory beyond 15 years, similar to Becker muscular dystrophy [BMD]) to severe (i.e., similar to Duchenne muscular dystrophy [DMD] with early requirement of a wheelchair), and cardiac involvement has been documented in some patients (40). In addition, other LGMD2 disorders (LGMD2C, 2D, 2E) are caused by sarcoglycan mutations $(\gamma-, \alpha$-, and $\beta$ sarcoglycan, respectively), suggesting that some LGMD phenotypes are sarcoglycanopathies. LGMDs may also include cardiac involvement associated with skeletal myopathy (41).

For these reasons, we have begun to analyze patients with DCM for mutations in genes encoding these proteins. In this report, we describe the identification of mutations in the $\delta$-sarcoglycan gene in familial and sporadic cases of pure DCM. This extends the spectrum of phenotypes seen with sarcoglycanopathies, now adding pure DCM to the potential clinical presentation.

\section{Methods}

Patient evaluation. All patients were evaluated by physical examination (particularly focused in the cardiac and neuromuscular systems), chest radiography, electrocardiography, and echocardiography. The left ventricular size and function was evaluated off M-mode and two-dimensional echocardiographic images as described previously (17-19). Cardiac catheterization, angiography and endomyocardial biopsy was performed in some patients based on clinical indication. After informed consent, blood for lymphoblastoid cell line immortalization and DNA extraction was obtained, as described previously $(17,18,21)$.

Control individuals. Two hundred unrelated and unaffected individuals (100 male, 100 female), as determined by history, physical examination, and echocardiography, were analyzed. After written informed consent, blood for lymphoblastoid cell line immortalization and DNA extraction was obtained.

Isolation and characterization of $\delta$-Sarcoglycan genomic clones. A human BAC DNA PCR pool library (Research Genetics, Huntsville, Alabama, USA) was screened by PCR using primers designed to amplify each of the exons containing the coding region. PCR was performed in a $10-\mu \mathrm{L}$ reaction containing $1.5 \mathrm{mM} \mathrm{MgCl}_{2}$, 10 pmol of each primer, 0.5 U Taq DNA polymerase (Life Technologies Inc., Rockville, Maryland, USA), using a Stratagene Robocyler (La Jolla, California, USA). After a 5-minute denaturation step at $94^{\circ} \mathrm{C}, 35$ rounds of amplification $\left(94^{\circ} \mathrm{C}\right.$ for 30 seconds, $54-60^{\circ} \mathrm{C}$ for 30 seconds, and $72^{\circ} \mathrm{C}$ for 20 seconds) were performed. This was followed by a $72^{\circ} \mathrm{C}$ incubation for 2 minutes.

The exons of $\delta$-sarcoglycan were subcloned into the vector pZero (Invitrogen, Carlsbad, California, USA). Clones were identified by whole cell PCR $(20 \mu \mathrm{L}$ reaction) and sequenced using an ABI373 (Perkin-Elmer Applied Biosystems, Foster City, California, USA) and Big Dye Terminator chemistry, according to the manufacturer's instructions.

Single-strand conformational polymorphism analysis. Mutation analysis was performed using a modification of the method of Orita et al. (42). PCR primers were designed to amplify across the entire human $\delta$ sarcoglycan gene in an exon-by-exon manner using the primers in Table 1 . Radioactive PCR was performed with $100 \mathrm{ng}$ genomic DNA in a $10 \mu \mathrm{L}$ reaction containing $1.5 \mathrm{mM} \mathrm{MgCl}_{2}, 10 \mathrm{pmol}$ of each primer, 0.05 $\mu \mathrm{Ci}\left[\alpha-{ }^{32} \mathrm{P}\right] \mathrm{dCTP}$ (Amersham Life Sciences Inc., Arlington Heights, Illinois, USA), 0.5 U Taq DNA polymerase, and 30 rounds of amplification $\left(92^{\circ} \mathrm{C}\right.$ for 30 seconds, $Y^{\circ} \mathrm{C}$ for 30 seconds, $72^{\circ} \mathrm{C}$ for 30 seconds), where $Y$ represents the annealing temperature shown in Table 1. After PCR amplification, the samples were denatured and then electrophoresed for 24 hours in a denaturing $10 \%$ polyacrylamide:bisacrylamide (50:1; Bio-Rad Laboratories Inc., Hercules, California, USA) gel with and without $12 \%$ glycerol, at $8 \mathrm{~W}$ in a $4^{\circ} \mathrm{C}$ cold room. Bands were visualized by exposure of the dried gels to Kodak BioMax MS-1 film (Eastman Kodak Co., Rochester, New York, USA).

DNA sequencing and protein structural analysis. Normal and aberrant single-strand conformational polymorphism (SSCP) conformers were cut directly from dried gels, eluted in $100 \mu \mathrm{L}$ of distilled water $\left(65^{\circ} \mathrm{C}\right.$ for 30 minutes), and then reamplified. After purification using Qiaquick columns (QIAGEN Inc., Valencia, California, USA), PCR products were sequenced, as already described here. Protein secondary structure predictions were performed using the GarnierOsguthorpe-Robson algorithm (43) with the Wisconsin Package (version 10.0), Genetics Computer Group (Madison, Wisconsin, USA).

Immunohistochemistry. Frozen myocardial sections (5$\mu \mathrm{m})$ were cut from the left and right ventricles. Unfixed sections were stained, using anti- $\alpha$-, anti- $\beta$-, anti- $\gamma$-, and anti- $\delta$-sarcoglycan antibodies, as well as antidystrophin (COOH-terminal antibody; Novocastra, Newcastle, United Kingdom). Each primary antibody was diluted 1:10 in PBS (pH 7.2) containing 5\% BSA and then added to the sections; these were incubated for 30 minutes at room temperature. The slides were washed 
three times in $0.1 \mathrm{X}$ PBS ( $\mathrm{pH} 7.2)$ at room temperature. The sections were then incubated with secondary antibody (FITC-conjugated anti-mouse; Novocastra), diluted 1:400 in PBS ( $\mathrm{pH} 7.2$ ) containing 5\% BSA, for 15 minutes at room temperature. The slides were washed three times in 0.1X PBS (pH 7.2) and mounted with Cytoseal 280 mounting medium (Stephens Scientific, Riverdale, New Jersey, USA) before observation.

\section{Results}

Phenotypic analysis: familial DCM. One family with familial DCM (FDCM no. 1100) was identified by history, physical examination, and echocardiography. All affected individuals were symptomatic with evidence of congestive heart failure at young age (Table 2). Neuromuscular examination was normal in all individuals, including muscle bulk and strength, reflexes, and gait. All affected individuals remained athletic until their onset of heart failure or sudden death. Echocardiographically, all affected individuals had severely dilated left ventricular chambers (with $Z$ scores $>4$ ) and moderate to severe systolic dysfunction (ejection fractions < 35\%). None of the clinically unaffected members of the family had abnormal echocardiograms. Cardiac transplantation was necessary in the 21-year-old son (III:7) of the proband (II:5) as seen in the pedigree (Figure 1). Before transplant, cardiac catheterization with angiography was performed. The coronary arteries were normal on selective angiography. Gross and histopathologic examination of the explanted heart demonstrated features of classic DCM, including increased heart weight, left ventricular dilatation, histopathologic evidence of myocyte hypertrophy, and moderate fibrosis. No evidence of ischemia was noted, and no inclusions were seen. The coronary arteries were normal by inspection and histologically. Creatine kinase (CK) was mildly elevated in this patient (Table 2), with the muscle isoform (CK$\mathrm{MM}$ ) entirely responsible for the elevation. No other affected members of the family had CK levels analyzed before death, and the unaffected members (II:2, II:3, III:3, III:4, and III:5) had normal levels. Autopsy specimens for deceased individuals were pathologically similar to those found in the explant of patient III:7, and the coronary arteries were normal in all cases.

Phenotypic analysis: sporadic DCM. Fifty patients ranging in age from 3 days to 18 years (mean, 9.5 years) with clinically apparent DCM presenting with heart failure and echocardiograms diagnostic for DCM were identified. Cardiac catheterization, angiography and endomyocardial biopsy was performed in 17 patients, and inflammatory infiltrate consistent with borderline $(n=4)$ or active $(n=2)$ myocarditis using the Dallas criteria was found in $35 \%$ of these individuals. The remaining patients were negative for inflammatory infiltrates. Coronary angiography was normal in all cases. In eight patients, cardiac transplantation was performed, whereas five patients died prematurely and without transplantation. Coronary arteries were nor- mal in all cases. CK levels were also normal in these individuals (Table 2).

Molecular analysis: genomic sequences. The cDNA sequence published by Nigro et al. (44) (GenBank accession no. X95191) and by Jung et al. (45). (GenBank accession no. U58331) identified alternative sequences at the $3^{\prime}$ end of the mRNA in expressed sequence tags (ESTs) identified in muscle (44) or in placenta (45). Subsequently, we refer to these spliced forms as the muscle (M) and placental (P) isoforms, respectively, although both are expressed in the heart (data not shown).

Screening of a human BAC library identified five BACs encoding the exons of $\delta$-sarcoglycan. BACs 523E7 and 417E23 encoded exons 2 and 3; BACs 555B9 and $557 \mathrm{H} 6$ encoded exons 4, 5, 6, and 7; and BAC 212M24 encoded exons 8 and 9. Subcloning of these BACs into plasmid vectors, followed by DNA sequence analysis, produced the sequences of the exon-intron boundaries, and primers complementary to intron sequences were synthesized for SSCP analysis (Table 1). Primers were designed to amplify across each of the exons including exon 9 of both the muscle isoform (DSarc9mF and D-Sarc9mR; Table 1) (44) and the placental isoform (D-Sarc9pF and D-Sarc9pR: Table 1) (45). Nigro et al. (44) reported the locations of seven introns within the coding region of human $\delta$-sarcoglycan mRNA. We identified an eighth intron located within the $5^{\prime}$ UTR. The location of this intron within the $5^{\prime}$ UTR suggests that the initiation codon is located within exon 2 , as occurs in the hamster.

\section{Molecular analysis: SSCP}

Familial DCM. SSCP was performed using the primers designed to amplify across the $\delta$-sarcoglycan gene. In family FDCM no. 1100, three affected individuals were iden-

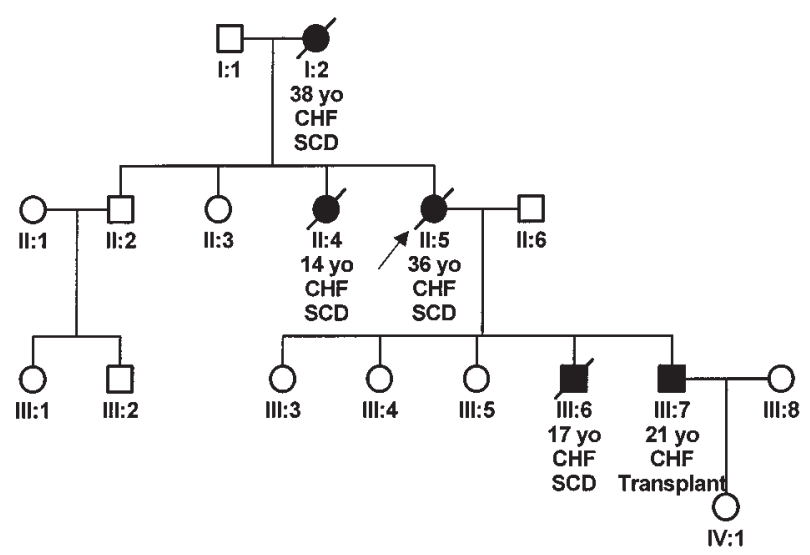

Figure 1

Familial DCM pedigree. Pedigree of family FDCM no. 1100. Note the severity of disease with early-onset $\mathrm{CHF}$ and sudden deaths in affected individuals. SCD, sudden cardiac death; CHF, congestive heart failure; yo, year old. Filled circles and squares represent affected females and males, respectively; open circles and squares represent unaffected females and males, respectively; diagonal line indicates death; and the arrow is the index case. 
Table 1

Oligonucleotide primers used for SSCP analysis of the human $\delta$-sarcoglycan gene

\begin{tabular}{|c|c|c|c|c|}
\hline Exon & Primer & $\begin{array}{l}\text { Primer sequence } \\
\qquad\left(5^{\prime}-3^{\prime}\right)\end{array}$ & $T_{\mathrm{A}}\left({ }^{\circ} \mathrm{C}\right)$ & $\begin{array}{l}\text { PCR product } \\
\text { size (bp) }\end{array}$ \\
\hline 2 & $\begin{array}{l}\text { D-Sarc2F } \\
\text { D-Sarc2R }\end{array}$ & $\begin{array}{l}\text { СCTGCCTTCTGGAAGTAATC } \\
\text { AAAATGACCATGAGCAGGGC }\end{array}$ & 60 & 229 \\
\hline 3 & $\begin{array}{l}\text { D-Sarc3F } \\
\text { D-Sarc3R }\end{array}$ & $\begin{array}{l}\text { TGCTTCTCTCTTGCCTCGTT } \\
\text { GCTAAACAAACCTAGATGGT }\end{array}$ & 50 & 243 \\
\hline 4 & $\begin{array}{l}\text { D-Sarc4F } \\
\text { D-Sarc4R }\end{array}$ & $\begin{array}{l}\text { TTACAGCCTGAGGTGTTTTG } \\
\text { GCAACAATAATGCCTCCTTC }\end{array}$ & 50 & 208 \\
\hline 5 & $\begin{array}{l}\text { D-Sarc5F } \\
\text { D-Sarc5R }\end{array}$ & $\begin{array}{l}\text { CCCCTTGGAGAGTTGTAATG } \\
\text { TATTCTGAGTGCCTCGCATG }\end{array}$ & 56 & 218 \\
\hline 6 & $\begin{array}{l}\text { D-Sarc6F } \\
\text { D-Sarc6R }\end{array}$ & $\begin{array}{l}\text { GATGAGACTAATGGTGTTTT } \\
\text { AAAATGTACACATGAGCATC }\end{array}$ & 52 & 244 \\
\hline 7 & $\begin{array}{l}\text { D-Sarc7F } \\
\text { D-Sarc7R }\end{array}$ & $\begin{array}{l}\text { CAGGTGACTCCAGTATCTCC } \\
\text { TGGCCAGTTGCACAGAGCAA }\end{array}$ & 50 & 188 \\
\hline 8 & $\begin{array}{l}\text { D-Sarc8F } \\
\text { D-Sarc8R }\end{array}$ & $\begin{array}{l}\text { AAAAGGGATCTTTATTGACG } \\
\text { TGTAGCTCTTTGAATTCTGT }\end{array}$ & 50 & 196 \\
\hline $9 m$ & $\begin{array}{l}\text { D-Sarc9mF } \\
\text { D-Sarc9mR }\end{array}$ & $\begin{array}{l}\text { CTGACCAATGCTTTCCTTCC } \\
\text { ATGCTGCCAACAATGTCCAC }\end{array}$ & 50 & 239 \\
\hline $9 p$ & $\begin{array}{l}\text { D-Sarc9pF } \\
\text { D-Sarc9pR }\end{array}$ & $\begin{array}{l}\text { AAGCTGGCAATATGGAAGCC } \\
\text { GGCTCCTTTTGTTGATACAC }\end{array}$ & 50 & 173 \\
\hline
\end{tabular}

$T_{\mathrm{A}}$ is the annealing temperature used in the PCR reactions. $9 \mathrm{~m}$ is exon 9 of the isoform identified by Nigro et al. (36) by sequencing an EST of muscle origin, whereas $9 p$ is exon 9 of the isoform sequenced by Jung et al. (45) from a placental EST.

tified with abnormal SSCP conformers (Figure 2). None of the unaffected members of the family and none of the 200 control individuals had this abnormal conformer. DNA sequence analysis identified a single nucleotide change, $451 T \rightarrow G$ in exon 6 , which changes the amino acid at codon 151 from serine to alanine (S151A). This change from a polar to nonpolar amino acid is significant and is predicted to alter the secondary structure of $\delta$-sarcoglycan, enabling an extended $\alpha$-helix. Computer modeling of this portion of the protein (43) supports secondary structural changes (data not shown) as well, but functional studies are required to confirm the effect of these mutations.

Sporadic DCM. SSCP analysis of the fifty sporadic cases of DCM identified four patients with abnormal conformers. In two patients, a 3-bp deletion (either 710-712delAGA or 711-713delGAA) of $\delta$-sarcoglycan exon $9 p$ was identified (Figure 3 ) that deletes a codon encoding the amino acid lysine at position 238 ( $\Delta \mathrm{K} 238)$; screening 200 control individuals, as well as the phenotypically normal parents of these children, did not identify the same abnormality. The deletion of lysine 238 is predicted to result in a change in the secondary structure in which the folding of the protein is disrupted, consistent with a disease-causing mutation. In addition, two other patients had abnormal conformers that, upon sequencing, appear to be polymorphisms. In one case, a $290 \mathrm{G} \rightarrow \mathrm{A}$ transition in exon 4 , which changes the arginine at codon 97 to glutamine (R97Q), was identified. This $290 \mathrm{G} \rightarrow \mathrm{A}$ transition was previously reported to be a polymorphism (45). In another patient, a $\mathrm{T} \rightarrow \mathrm{A}$ transversion within intron 4 , 38 nucleotides from the intron 4-exon 5 boundary, was found. One of 200 controls was identified with the identical change, most likely representing a polymorphism, especially considering the distance from the intron-exon boundary. However, in an attempt to document whether this caused a change in the splicing of the mRNA, RNA was isolated from lymphoblastoid cell lines (from the patient and from a control) and subjected to RT-PCR using primers designed to amplify this region. However, amplification was unsuccessful, probably due to limited illegitimate transcription of $\delta$ sarcoglycan in lymphoblasts.

Immunohistochemistry. Tissue from the explanted heart of one of the patients with the $\Delta \mathrm{K} 238$ deletion was available for immunohistochemical analysis. Frozen sections of right and left ventricle were stained with antibodies against $\alpha$-, $\beta$-, $\gamma$-, and $\delta$-sarcoglycan, or the $\mathrm{COOH}$-terminus of dystrophin. In parallel, myocardial sections from the explanted heart of a patient with congenital heart disease (i.e., control patient) were stained for comparison. Although the staining of dystrophin and $\alpha-, \beta$ (data not shown), and $\gamma$-sarcoglycan (Figure 4) was indistinguishable between the control and affected patient samples, the staining for $\delta$-sarcoglycan was significantly reduced in the patient with the $\Delta \mathrm{K} 238$ mutation (Figure 4). Interestingly the $\delta$-sarcoglycan antibody is $\mathrm{NH}_{2}$-terminal specific whereas the mutation is $\mathrm{COOH}$-terminal, suggesting this mutation leads to a significant change in $\delta$-sarcoglycan structure.

\section{Discussion}

Although the pathophysiology of DCM is well known (5), the underlying genetic mechanism for this disorder has remained unclear. Whether the disease results from

a

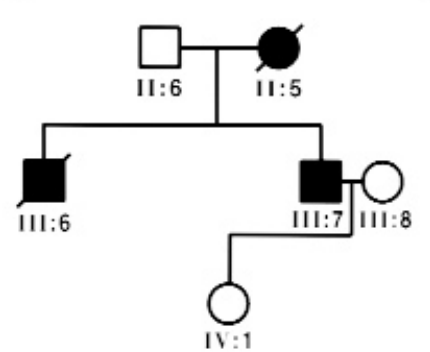

Normal
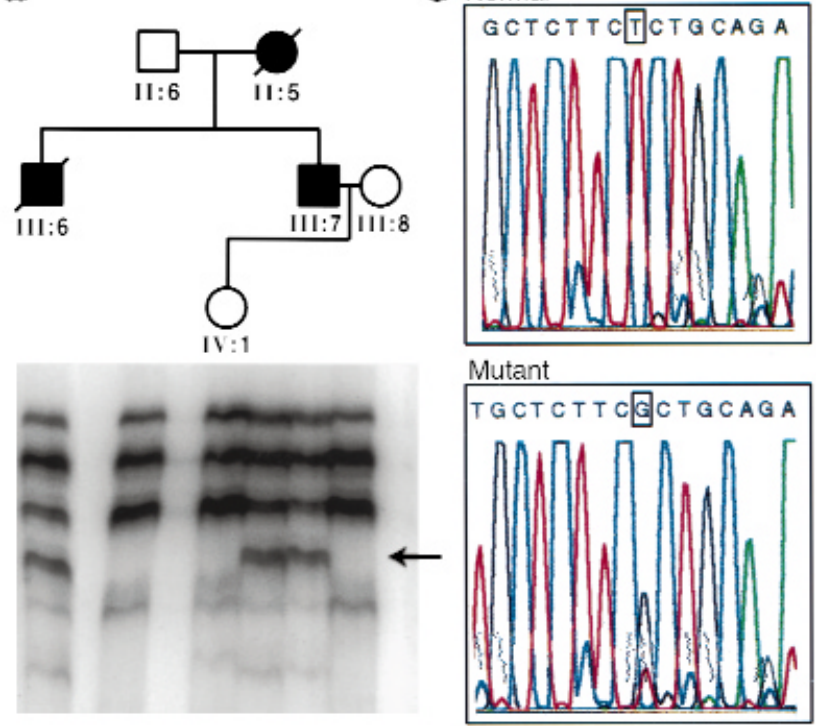

Figure 2

Mutation analysis of $\delta$-sarcoglycan in familial DCM. (a) SSCP performed using primers for exon 6 of $\delta$-sarcoglycan identifies an abnormal conformer in affected individuals only (arrow). (b) Sequence analysis demonstrates a $T \rightarrow G$ substitution at position 151 , which changes the wild-type serine to alanine (S151A). 
Table 2

Clinical characteristics of patients

\begin{tabular}{lcccc}
\hline Patient & Clinical presentation & LVEDD (Z score) & EF (Z score) & CK (MM\%) \\
FDCM no. 1100 & & & \\
I:2 & CHF, SCD, age 38y & ND & ND & ND \\
II:4 & CHF, SCD, age 14y & ND & ND & ND \\
II:5 & CHF, SCD, age 36y & $62 \mathrm{~mm}(5.1)$ & $26 \%(6.0)$ & ND \\
III:6 & CHF, SCD, age 17y & $68 \mathrm{~mm}(4.0)$ & $29 \%(5.6)$ & ND \\
III:7 & CHF, transplant, age 21 y & $70 \mathrm{~mm}(4.5)$ & $20 \%(7.1)$ & $350(100 \%)$ \\
Sporadics & & & & \\
FDCM-36 & CHF, age 9 mo. & $40 \mathrm{~mm}(8.5)$ & $15 \%(8.0)$ & $50(100 \%)$ \\
FDCM-48 & CHF, age 14y & $58 \mathrm{~mm}(4.8)$ & $32 \%(4.3)$ & $35(100 \%)$
\end{tabular}

$\mathrm{CHF}$, congestive heart failure; SCD, sudden cardiac death; LVEDD, left ventricular end diastolic dimension; Z score, standard deviation; EF, ejection fraction; CK, creatine kinase; CK (MM\%), creatine kinase muscle isoform percent; ND, not determined.

inflammation, autoimmune, or genetic causes has been speculated upon for decades (46) but a unifying mechanism has not been proved. During the past several years, however, a variety of clues to the underlying cause of DCM, as well as the underlying basis for other inherited cardiovascular diseases, have emerged. This has led us to speculate on the existence of a final common pathway of cardiovascular disease in which specific phenotypes result from mutations in families of proteins essential for specific functions. For instance, the basis for hypertrophic cardiomyopathy (HCM), a primary heart muscle disease in which ventricular wall thickening (hypertrophy) and diastolic dysfunction occur, has been demonstrated to be due to mutations in genes encoding sarcomeric proteins such as $\beta$ myosin heavy chain, $\alpha$-tropomyosin, cardiac troponin $\mathrm{T}$, cardiac troponin I, myosin binding protein-C, essential and regulatory myosin light chains (reviewed in ref. 31 ), and cardiac actin (47). In addition, the inherited long QT syndromes (LQTS) have been shown to be due to mutations in genes encoding ion channels, such as the potassium channel genes KVLQT1, KCNE1, and HERG, KCNE2, and the cardiac sodium channel gene SCN5A (reviewed in ref. 32). Owing to the consistent protein classes found to be mutated in phenotypically similar patients (i.e., the final common pathways of sarcomeric proteins in HCM; ion channels in LQTS), we used this final common pathway hypothesis $(29,30$, 48) to speculate that similar protein types would be mutated in DCM as well.

Five genes have been previously identified and characterized in cases of familial DCM. In Barth syndrome, the gene 64.5 , which encodes a novel protein family called tafazzins, is mutated (11-14). Although well characterized at the molecular level, to our knowledge the function of the encoded protein is not currently known. However, the gene responsible for XLCM, dystrophin, is well defined (15-17). This gene, which also causes Duchenne (DMD) and Becker (BMD) muscular dystrophy when mutated (49), encodes a large $(427-\mathrm{kDa})$ cytoskeletal protein that resides at the inner face of the sarcolemma, colocalizing with $\beta$-spectrin and vinculin. Dystrophin protein is thought to assume a rod-shaped structure with an actin-binding domain at the $\mathrm{NH}_{2}$-terminus. The $\mathrm{COOH}$-terminal domain is associated with a large transmembrane glycoprotein complex, the DAG, which is thought to mechanically stabilize the plasma membrane of muscle cells as well as serving a cell signaling role. This complex is formed by the dystroglycan subcomplex $(\alpha$-dystroglycan and $\beta$-dystroglycan), sarcoglycan subcomplex $(\alpha-, \beta-, \gamma-$, and $\delta$ sarcoglycan), caveolin-3, syntrophin, dystrobrevin, and sarcospan and serves as a link between cytoplasmic actin, the membrane, and the extracellular matrix of muscle via laminin- $\alpha 2(50,51)$. Mutations in $d y s-$ trophin, the DAG subcomplexes, or laminin result in a wide spectrum of skeletal myopathy and/or cardiomyopathy in humans and animal models such as the mouse or hamster $(30-36,52)$.

The third mutant gene thus far identified, cardiac actin, has been identified as the gene responsible for the chromosome 15q14-linked autosomal dominant FDCM (22). This gene encodes a member of the thin filament of the sarcomeric unit. When mutated near the dystrophin-binding site, DCM occurs. However, when mutations near the sarcomeric end of actin occur, a

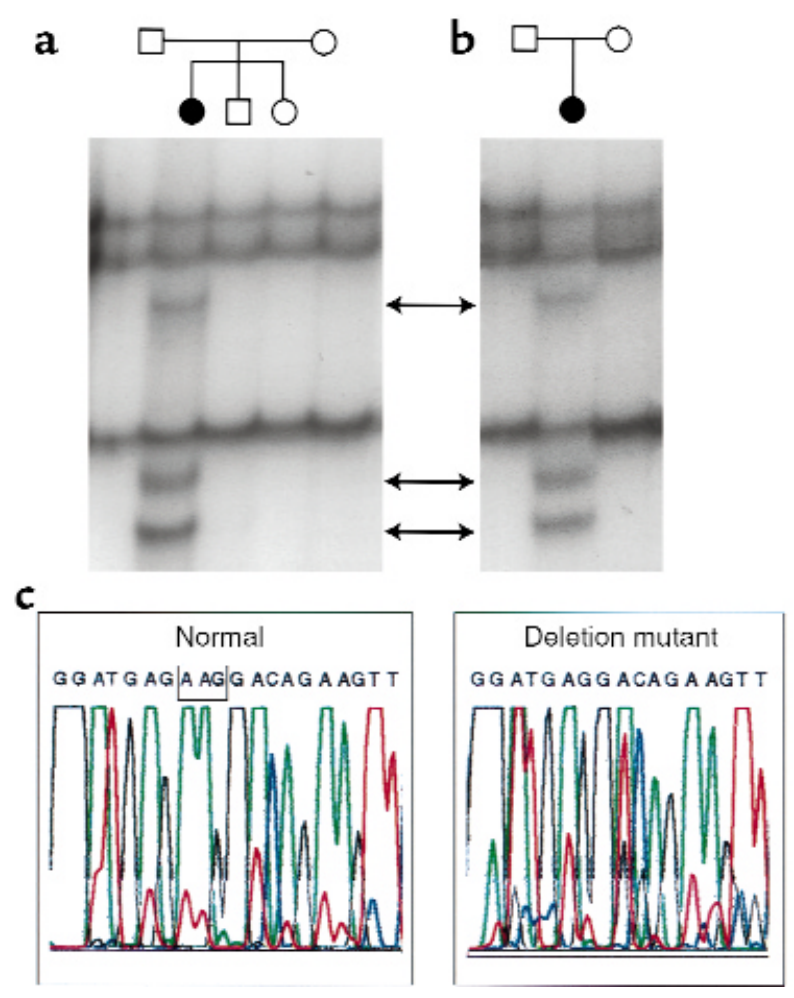

Figure 3

Mutation analysis of $\delta$-sarcoglycan in sporadic DCM. ( $\mathbf{a}$ and $\mathbf{b})$ Abnormal SSCP conformers in two individuals with sporadic DCM. (c) Sequence analysis identifies a 3-bp deletion in exon $9 p$ that deletes the codon for the amino acid lysine at position 238 ( $\Delta K 238)$. 


\section{Figure 4}

Immunohistochemical analysis of the DAC complex and dystrophin. The detection of $\delta$-sarcoglycan (a) and $\gamma$-sarcoglycan (b) in the myocardium of the patient with the $\Delta \mathrm{K} 238$ mutation of $\delta$-sarcoglycan. Note the reduction in the intensity of staining of $\delta$ sarcoglycan, whereas $\gamma$-sarcoglycan remains the same as the control.
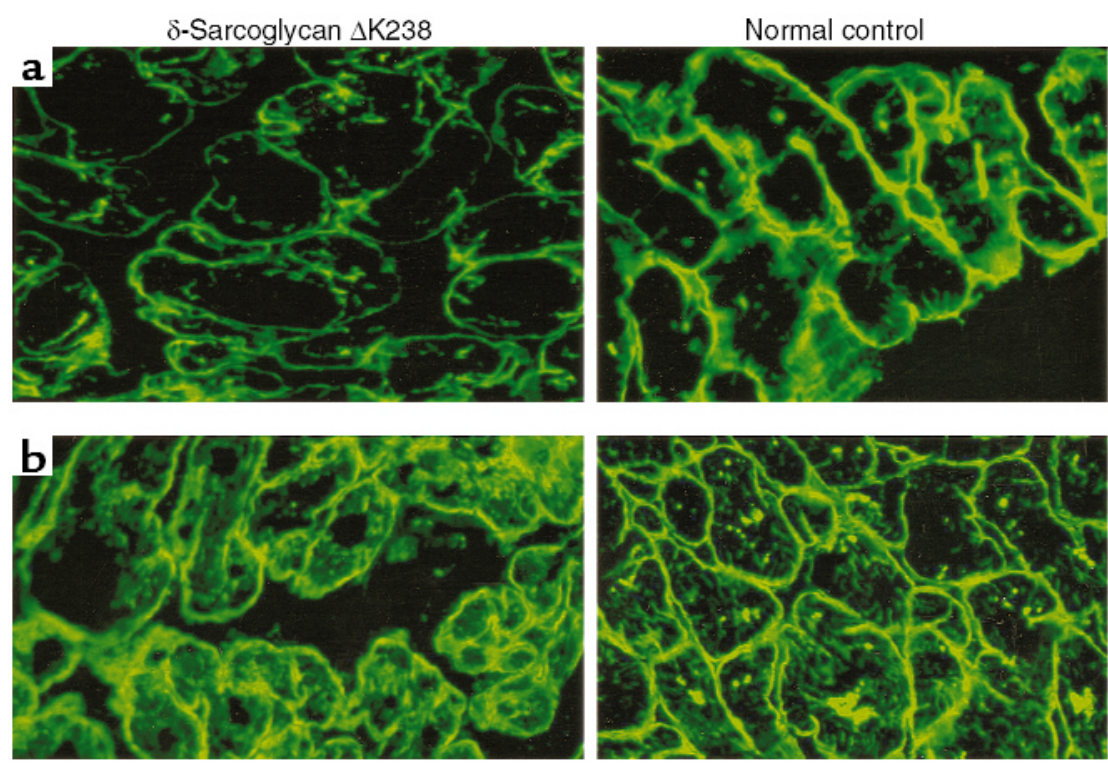

HCM phenotype develops (46). The likely reason that this mutant gene causes a DCM phenotype is that the link between dystrophin and actin is disrupted, thereby disassociating the actin cytoskeleton from the muscle membrane and extracellular matrix, leading to cellular degeneration and necrosis. Recently, mutations in $\alpha$ actin were shown to cause the skeletal muscle disorders actin myopathy and nemaline myopathy as well (53).

The other two genes identified in FDCM are desmin (2q35) (ref. 25) and lamin A/C (1p1-1q21) (ref. 28), both of which are thought to cause abnormalities of structural support when mutated, as well. Interestingly, these genes have both been shown to be associated with skeletal myopathy and, in some cases, with conduction system disease $(28,54,55)$. The identification of mutations in $\delta$-sarcoglycan, another member of the cytoskeleton/sarcolemmal structural support, further supports our final common pathway hypothesis $(29,30,48)$. Furthermore, mutations in this gene have previously been shown to cause either dilated or hypertrophic cardiomyopathies in the hamster and mouse, providing support that this gene, which maps to chromosome 5q33-34 in humans (42), is disease-causing in the patients described in this report.

LGMDs are a group of disorders that are characterized by progressive muscle weakness affecting both upper and lower limbs. They mainly have autosomal recessive inheritance, elevated $\mathrm{CK}$, and are due to mutations in sarcoglycans, including LGMD2C $(\gamma-$ sarcoglycan), LGMD2D ( $\alpha$-sarcoglycan), LGMD2E $(\beta$ sarcoglycan), and LGMD2F ( $\delta$-sarcoglycan). LGMD2F was initially reported by Nigro et al. (56) in four Brazilian families, and all affected members had a frameshift mutation in exon $7(\Delta 656 \mathrm{C})$ that resulted in premature truncation of the translatable $\delta$-sarcoglycan protein. These patients had severe DMD-like disease. Duggan et al. (57) and Moreira et al. (40) identified homozygous mutations (W30X and R165X nonsense mutations, and E262K missense mutation, respectively) in $\delta$-sarcoglycan in patients with DMDlike disease as well. In the case of the family studied by Moreira et al., the electrocardiogram was consistent with left ventricular hypertrophy, but no other cardiac information (i.e., echocardiogram) was reported.

Thus, LGMD associated with sarcoglycan mutations appears to be inherited as a recessive disease. However, in the patients with DCM described in this report, we propose that dominant negative mutations in $\delta$-sarcoglycan result in absent skeletal myopathy in the face of late-onset DCM. These mutations are similar to those described in LGMD2F, except that they are heterozygous. Although heterozygotes within LGMD2F families have not been described to be affected, it is possible that cardiac evaluation overlooked cardiac pathology or the cardiac dysfunction had not become apparent at the time of evaluation, given that DCM is, in many instances, age dependent. In DMD and BMD, disorders of dystrophin, female carriers commonly develop cardiomyopathy but usually do so later in life than the affected males. Further, as cardiac disease is now appearing to be common in most (if not all) skeletal myopathies, it is not surprising that $\delta$-sarcoglycan mutations could have wide clinical phenotypic heterogeneity. Melacini et al. (41) reported DCM to be relatively common in sarcoglycanopathies (about 30\%), although they did not find DCM with $\delta$-sarcoglycan mutations in the LGMD patients with DCM studied.

Interestingly Nigro et al. (44) identified a dominant missense mutation (N211Y) in $\delta$-sarcoglycan in a family with mild proximal myopathy and mild CK elevations (threefold). Four autosomal dominant forms of LGMD have also been described (LGMD1A-D). To our knowledge, only genes for LGMD1B, which is associated with atrioventricular conduction disturbances, and LGMD1C have been identified, namely, lamin A/C (58) and caveolin-3 (59), respectively. It is 
possible that mutations in different domains of $\delta$ sarcoglycan result in either a cardiac or skeletal muscle phenotype, similar to that described for dystrophin $(15-17,49)$. Similarly, these differences could account for the modes of inheritance (i.e., autosomal recessive or autosomal dominant).

Multiple animal models with sarcoglycan deficiency have been produced or are naturally occurring, including the cardiomyopathic hamster due to deletion of exons 1 and 2 of $\delta$-sarcoglycan (35). Hack et al. (60) generated a $\gamma$-sarcoglycan-deficient mouse that developed progressive muscular dystrophy, pronounced cardiac muscle degeneration, and reduced survival. These mice also had reduced levels of $\beta$ - and $\delta$-sarcoglycan staining of muscle (with normal dystrophin, dystroglycan, and laminin- $\alpha 2$ ) and elevated CK at baseline (suggesting CK release occurs by mechanisms other than mechanical injury) and with exercise. Coral-Vazquez et al. (61) studied mice deficient in $\alpha$-sarcoglycan and $\delta$-sarcoglycan and demonstrated that only $\delta$-sarcoglycan null mice developed cardiomyopathy, with focal areas of necrosis by 3 months of age; death occurred by 6 months of age typically. Coronary artery abnormalities, particularly constriction with pre- and poststenotic dilation and reduction in lumen size, were noted. This has been supported in studies in humans (62), but no coronary artery disease has been found in any of the patients described here. Coronary angiography and echocardiography (transthoracic and in some cases transesophageal) identified normal coronary arteries in all patients with $\delta$-sarcoglycan mutations, and no evidence of ischemia was found in the myocardium. Histologically and by gross evaluation, the coronary arteries were normal in the explanted hearts of the transplanted sporadic and familial cases. Araishi et al. (63) studied $\beta$-sarcoglycan-deficient mice, which exhibited progressive muscular dystrophy, muscular hypertrophy, severe elevations in CK (about 100 times higher than in wild type), and cardiac fibrosis. Immunohistochemical staining demonstrated $\alpha$-, $\beta$-, $\gamma$-, and $\delta$-sarcoglycan and sarcospan to be absent in the sarcolemma, whereas laminin- $\alpha 2, \alpha$ - and $\beta$-dystroglycan, and dystrophin were normal. In one patient with a $\Delta \mathrm{K} 238$ deletion, no reduction in $\alpha$-, $\beta$-, or $\gamma$-sarcoglycan or dystrophin, was noted.

In conclusion, the description of mutations in $\delta$ sarcoglycan in patients with DCM provides further support for the concept that the final common pathway for DCM is the cytoarchitecture, comprising the cytoskeleton, sarcolemma, and interacting components. In addition, the fact that mutations in $\delta$-sarcoglycan and dystrophin, as well as mutations in G4.5, can also result in skeletal myopathy, suggests that patients with DCM should be carefully evaluated for skeletal muscle weakness and that neurologists caring for patients with skeletal myopathies should be cognizant of the potential for associated cardiomyopathies in their patients.

\section{Acknowledgments}

We thank P. Sen of the Child Health Research Center, Baylor College of Medicine, for the DNA sequence analysis. K. Bowles is a Howard Hughes Medical Institute predoctoral fellow. This work was supported by the Abercrombie Cardiology Fund of Texas Children's Hospital (N.E. Bowles), Texas Children's Hospital Foundation Chair in Pediatric Cardiac Research (J.A. Towbin) and NIH grants from the National Heart, Lung, and Blood Institute (J.A. Towbin).

1. Dec, G.W., and Fuster, V. 1994. Idiopathic dilated cardiomyopathy. N. Engl. J. Med. 331:1564-1575.

2. Manolio, T.A., Baughman, K.L., Rodeheffer, R., Pearson, T.A., and Bristow, J.D. 1992. Prevalence and etiology of idiopathic dilated cardiomyopathy (summary of a National Heart, Lung, and Blood Institute Workshop). Am. J. Cardiol. 69:1458-1466.

3. Codd, M.B., Sugrue, D.D., Gersh, B.J., and Melton, L.J. 1989. Epidemiology of idiopathic dilated and hypertrophic cardiomyopathy. A population-based study of Olmsted County, Minnesota, 1975-1984. Circulation. 80:564-572.

4. O'Connell, J.B., and Bristow, M.R. 1994. Economic impact of heart failure in the United States: time for a different approach. J. Heart Lung Transplant. 13:S107-S112.

5. Kasper, E.K., et al. 1994. The causes of dilated cardiomyopathy: a clinicopathologic review of 673 consecutive patients. J. Am. Coll. Cardiol. 23:586-590.

6. Towbin, J.A. 1999. Pediatric myocardial disease. Pediatr. Clin. North. Am. 46:289-312.

7. Michels, V.V., et al. 1992. The frequency of familial dilated cardiomyopathy in a series of patients with idiopathic dilated cardiomyopathy. $N$. Engl. J. Med. 326:77-82.

8. Keeling, P.J., et al. 1995. Familial dilated cardiomyopathy in the United Kingdom. Br. Heart J. 73:417-421.

9. Grunig, E., et al. 1998. Frequency and phenotypes of familial dilated cardiomyopathy. J. Am. Coll. Cardiol. 31:186-194.

10. Towbin, J.A. 1993. Molecular genetic aspects of cardiomyopathy. Biochem. Med. Metab. Biol. 49:285-300.

11. Bione, S., et al. 1996. A novel X-linked gene, G4.5. is responsible for Barth syndrome. Nat. Genet. 12:385-389.

12. Johnston, J., et al. 1997. Mutation characterization and genotype-phenotype correlation in Barth syndrome. Am. J. Hum. Genet. 61:1053-1058.

13. D'Adamo, P., et al. 1997. The X-linked gene G4.5 is responsible for different infantile dilated cardiomyopathies. Am. J. Hum. Genet. 61:862-867.

14. Bleyl, S.B., et al. 1997. Neonatal, lethal noncompaction of the left ventricular myocardium is allelic with Barth syndrome. Am. J. Hum Genet. 61:868-872.

15. Towbin, J.A., et al. 1993. X-linked dilated cardiomyopathy: molecular genetic evidence of linkage to the Duchenne muscular dystrophy (dystrophin) gene at the Xp21 locus. Circulation. 87:1854-1865.

16. Muntoni, F., et al. 1993. Brief report: deletion of the dystrophin musclespecific promoter region associated with X-linked dilated cardiomyopathy. N. Engl. J. Med. 329:921-925.

17. Ortiz-Lopez, R., Li, H., Su, J., Goytia, V., and Towbin, J.A. 1997. Evidence for a dystrophin missense mutation as a cause of X-linked dilated cardiomyopathy. Circulation. 95:2434-2440.

18. Durand, J.B., et al. 1995. Localization of a gene responsible for familial dilated cardiomyopathy to chromosome 1q32. Circulation. 92:3387-3389.

19. Siu, B.L., et al. 1999. Familial dilated cardiomyopathy locus maps to chromosome 2q31. Circulation. 99:1022-1026.

20. Krajinovic, M., et al. 1995. Linkage of familial dilated cardiomyopathy to chromosome 9. Am. J. Hum. Genet. 57:846-852.

21. Bowles, K.R., et al. 1996. Gene mapping of familial autosomal dominant dilated cardiomyopathy to chromosome 10q21-23. J. Clin. Invest. 98:1355-1360.

22. Olson, T.M., Michels, V.V., Thibodeau, S.N., Tai, Y.S., and Keating, M.T. 1998. Actin mutations in dilated cardiomyopathy, a heritable form of heart failure. Science. 280:750-752.

23. Kass, S., et al. 1994. A genetic defect that causes conduction system disease and dilated cardiomyopathy maps to $1 \mathrm{p} 1-1 \mathrm{q} 1$. Nat. Genet. 7:546-551.

24. Jung, M., et al. 1999. Investigation of a family with autosomal dominant dilated cardiomyopathy defines a novel locus on chromosome 2q14-q22. Am. J. Hum. Genet. 65:1068-1077.

25. Li, D., et al. 1999. Desmin mutation responsible for idiopathic dilated cardiomyopathy. Circulation. 100:461-464.

26. Olson, T.M., and Keating, M.T. 1996. Mapping a cardiomyopathy locus to chromosome 3p22-p25. J. Clin. Invest. 97:528-532. 
27. Messina, D.N., Speer, M.C., Pericak-Vance, M.A., and McNally, E.M. 1997. Linkage of familial dilated cardiomyopathy with conduction defect and muscular dystrophy to chromosome 6q23. Am. J. Hum. Genet. 61:909-917.

28. Fatkin, D., et al. 1999. Missense mutations in the rod domain of the lamin A/C gene as causes of dilated cardiomyopathy and conduction system disease. N. Engl. J. Med. 341:1715-1724.

29. Towbin, J.A., Bowles, K.R., and Bowles, N.E. 1999. Etiologies of cardiomyopathy and heart failure. Nat. Med. 5:266-267.

30. Towbin, J.A. 1998. The role of cytoskeletal proteins in cardiomyopathy. Curr. Opin. Cell Biol. 10:131-139.

31. Bonne, G., Carrier, L., Richard, P., Hainque, B., and Schwartz, K. 1998. Familial hypertrophic cardiomyopathy: from mutations to functional defects. Circ. Res. 83:580-593.

32. Wang, Q., Bowles, N.E., and Towbin, J.A. 1998. The molecular basis of long QT syndrome and prospects for therapy. Mol. Med. Today. 4:382-388.

33. Bajusz, E., Baker, J.R., Nixon, C.W., and Homburger, F. 1969. Spontaneous, hereditary myocardial degeneration and congestive heart failure in a strain of Syrian hamsters. Ann. NY Acad. Sci. 156:105-129.

34. Homburger, F. 1979. Myopathy of hamster dystrophy: history and morphologic aspects. Ann. NY Acad. Sci. 317:1-17.

35. Sakamoto A., et al. 1997. Both hypertrophic and dilated cardiomyopathies are caused by mutation of the same gene, $\delta$-sarcoglycan, in hamster: an animal model of disrupted dystrophin-associated glycoprotein complex. Proc. Natl. Acad. Sci. USA. 94:13873-13878.

36. Nigro, V., et al. 1997. Identification of the Syrian hamster cardiomyopathy gene. Hum. Mol. Genet. 6:601-607.

37. Sakamoto, A., Abe, M., and Masaki, T. 1999. Delineation of genomic deletion in cardiomyopathic hamster. FEBS Lett. 447:124-128.

38. Straub, V. 1998. Molecular pathogenesis of muscle degeneration in the delta-sarcoglycan-deficient hamster. Am. J. Pathol. 153:1623-1630.

39. Nigro, V., et al. 1996. Autosomal recessive limb-girdle muscular dystrophy, LGMD2F, is caused by a mutation in the delta-sarcoglycan gene. Nat. Genet. 14:195-198.

40. Moreira, E.S., et al. 1998. A first missense mutation in the delta sarcoglycan gene associated with a severe phenotype and frequency of limbgirdle muscular dystrophy type 2F (LGMD2F) in Brazilian sarcoglycanopathies. J. Med. Genet. 35:951-953.

41. Melacini, P., et al. 1999. Heart involvement in muscular dystrophies due to sarcoglycan gene mutations. Muscle Nerve. 22:473-479.

42. Orita, M., Iwahana, H., Kanazawa, H., Hayashi, K., and Sekiya, T. 1989. Detection of polymorphisms of human DNA by gel electrophoresis as single-strand conformation polymorphisms. Proc. Natl. Acad. Sci. USA. 86:2766-2770.

43. Garnier, J., Osguthorpe, D.J., and Robson, B. 1978. Analyses of the accuracy and implications of simple methods for predicting the secondary structure of globular proteins. J. Biol. Chem. 120:97-120.

44. Nigro, V., et al. 1996. Identification of a novel sarcoglycan gene at 5q33 encoding a sarcolemmal $35 \mathrm{kDa}$ glycoprotein. Hum. Mol. Genet. 5:1179-1186.

45. Jung, D., et al. 1996. Characterization of delta-sarcoglycan, a novel com- ponent of the oligomeric sarcoglycan complex involved in limb-girdle muscular dystrophy. J. Biol. Chem. 271:32321-32329.

46. Bender, J.R. 1991. Idiopathic dilated cardiomyopathy. An immunologic, genetic, or infectious disease, or all of the above? Circulation. 83:515-522.

47. Mogensen, J., et al. 1999. $\alpha$-cardiac actin is a novel gene in familial hypertrophic cardiomyopathy. J. Clin. Invest. 103:R39-R43.

48. Towbin, J.A. 1999. Towards an understanding of the cause of mitral valve prolapse. Am. J. Hum. Genet. 65:1238-1241.

49. Hoffman, E.P., Brown, R.H., and Kunkel, L.M. 1987. Dystrophin: the protein product of the Duchenne muscular dystrophy locus. Cell. 51:919-928.

50. Lim, L.E., and Campbell, K.P. 1998. The sarcoglycan complex in limbgirdle muscular dystrophy. Curr. Opin. Neurol. 11:443-452.

51. Matsumura, K., et al. 1999. Sarcoglycan complex: a muscular supporter of dystroglycan-dystrophin interplay? Cell. Mol. Biol. 45:751-762.

52. Towbin, J.A. 1995. Biochemical and molecular characterization of Xlinked dilated cardiomyopathy (XLCM). In Developmental mechanisms of heart disease. E.B. Clark, R.R. Markwald, and A. Takao, editors. Futura Publishing Co. New York, New York, USA. 121-132.

53. Nowak, K.J., et al. 1999. Mutations in the skeletal muscle alpha-actin gene in patients with actin myopathy and nemaline myopathy. Nat. Genet. 23:208-212.

54. Bonne, G., et al. 1999. Mutations in the gene encoding lamin A/C cause autosomal dominant Emery-Dreifuss muscular dystrophy. Nat. Genet. 21:285-288.

55. Raffaele Di Barletta, M., et al. 2000. Different mutations in the LMNA gene cause autosomal dominant and autosomal recessive Emery-Dreifuss muscular dystrophy. Am. J. Hum. Genet. 66:1407-1412.

56. Nigro, V., et al. 1996. Autosomal recessive limb-girdle muscular dystrophy, LGMD2F, is caused by a mutation in the delta-sarcoglycan gene. Nat. Genet. 14:195-198.

57. Duggan, D.J., et al. 1997. Mutations in the delta-sarcoglycan gene are a rare cause of autosomal recessive limb-girdle muscular dystrophy (LGMD2). Neurogenetics. 1:49-58.

58. Muchir, A., et al. 2000. Identification of mutations in the gene encoding lamins $\mathrm{A} / \mathrm{C}$ in autosomal dominant limb girdle muscular dystrophy with atrioventricular conduction disturbances (LGMD1B). Hum. Mol. Genet. 9:1453-1459.

59. Minetti, C., et al. 1998. Mutations in the caveolin-3 gene cause autosomal dominant limb-girdle muscular dystrophy. Nat. Genet. 18:365-368.

60. Hack, A.A., et al. 1998. Gamma-sarcoglycan deficiency leads to muscle membrane defects and apoptosis independent of dystrophin.J. Cell. Biol. 142:1279-1287.

61. Coral-Vazquez, R, et al. 1999. Disruption of the sarcoglycan-sarcospan complex in vascular smooth muscle: a novel mechanism for cardiomyopathy and muscular dystrophy. Cell. 98:465-474.

62. Gnecchi-Ruscone, T., et al. 1999. Cardiomyopathy in Duchenne, Becker, and sarcoglycanopathies: a role for coronary dysfunction? Muscle Nerve. 22:1549-1556.

63. Araishi, K., et al. 1999. Loss of the sarcoglycan complex and sarcospan leads to muscular dystrophy in beta-sarcoglycan-deficient mice. Hum. Mol. Genet. 8:1589-1598. 